\title{
The accumulated experience of targeted temperature management is associated with improved outcomes in cardiac arrest survivors without temperature management
}

\author{
Dean-An Ling, MD; Chien-Hua Huang, MD, PhD; Wei-Tien Chang, MD, PhD;
} Wei-Ting Chen, MD; Wen-Jone Chen, MD, PhD; Min-Shan Tsai, MD, PhD

Department of Emergency Medicine, National Taiwan University Medical College and Hospital, Taipei, Taiwan

\section{Background}

Targeted temperature management (TTM) has been proved to improve the survival and neurological outcome in cardiac arrest survivors and incorporated as part of post-cardiac arrest care as indicated. However, whether the accumulation of TTM experience also benefits patients not receiving TTM has not been investigated yet.

\section{Objectives}

To evaluate the influence of TTM experience on survival and neurological outcome in adult non-traumatic cardiac arrest survivors not receiving TTM.

\section{Methods}

Non-traumatic adult ( $\geq 18$ years-old) cardiac arrest victims who survived to intensive care unit admission were retrospectively recruited from a single tertiary medical center during the period from 2006 to 2009 , and 2011 to 2017 . This university hospital is a tertiary referred center hospital with about 100,000 ED visits per year. The development of the TTM protocol in this hospital was shown in Figure 1.

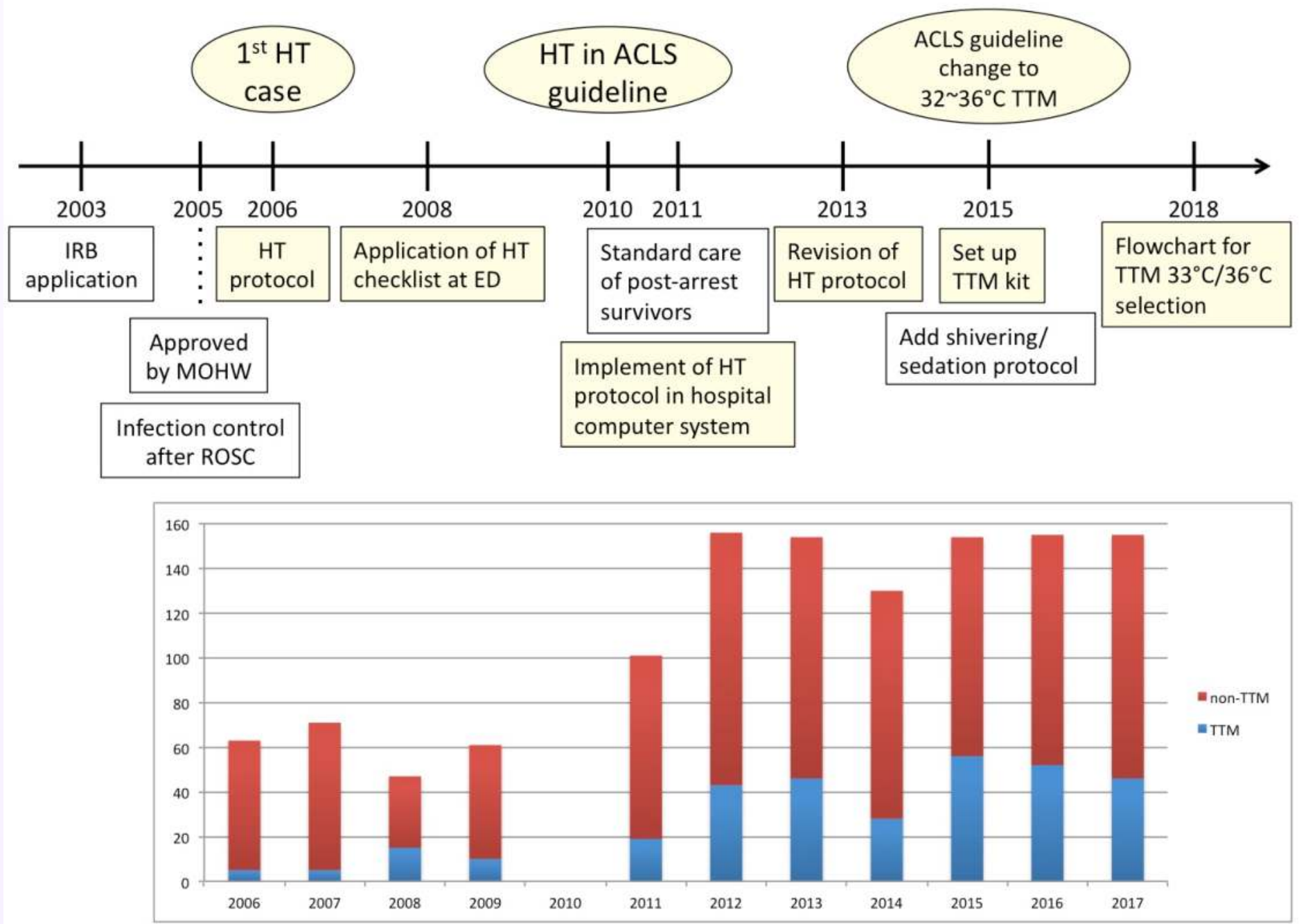

Figure 1. The development of TTM protocol in NTUH. HT: Hypothermia

The inclusion and exclusion flowchart was illustrated in Figure 2. A total of 1247 patients were enrolled. Patient who received TTM $(n=325)$ were excluded. Non-TTM patients $(n=922)$ were retrieved for further analysis. The TTM accumulated experience was defined as the sum of TTM cases and categorized by year. The association between TTM accumulated experience and outcomes per year in the non-TTM cardiac arrest survivors were evaluated.

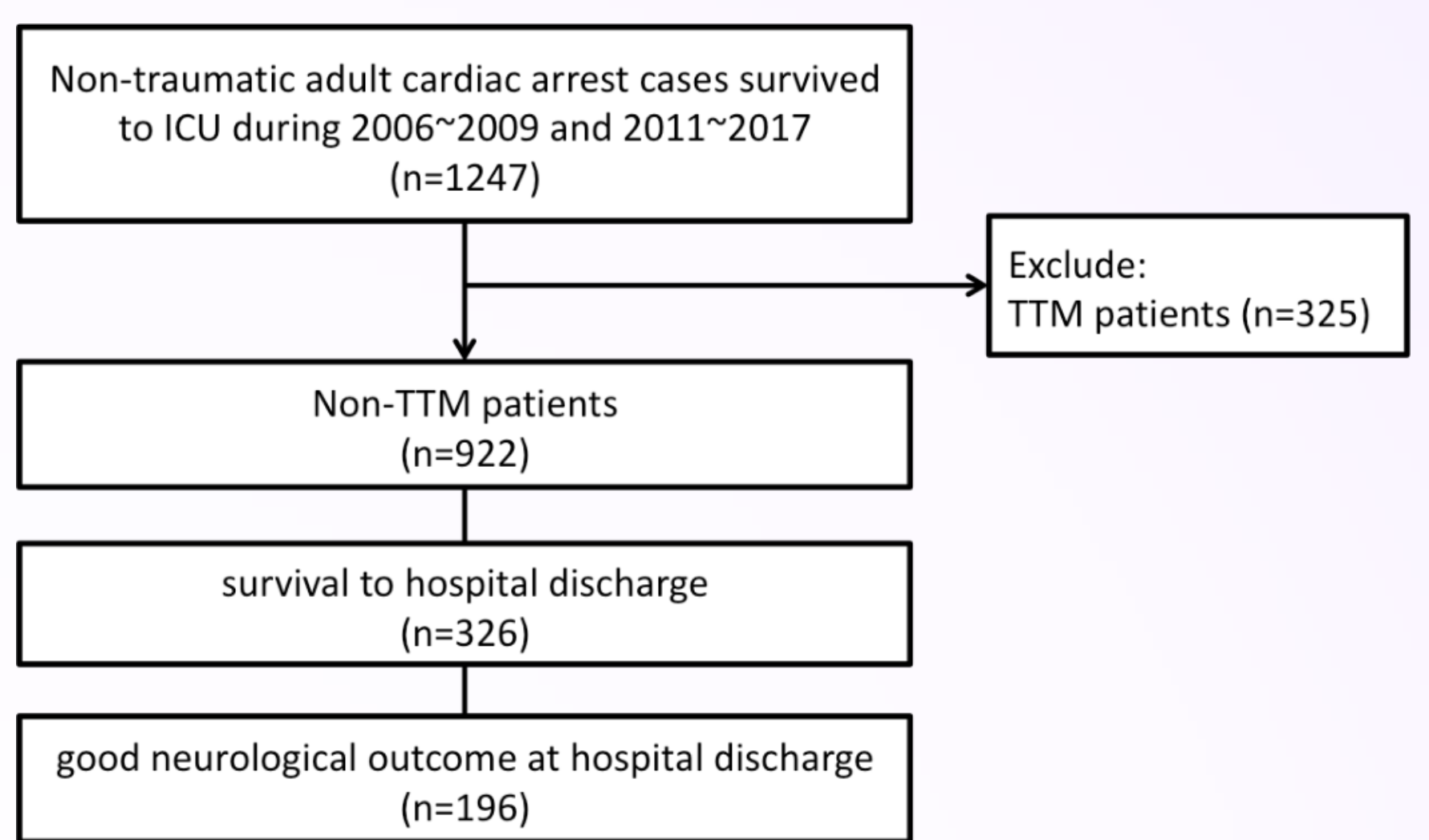

Figure 2. Inclusion and exclusion flowchart.

\section{Results}

For the adult non-traumatic cardiac arrest patients who did not received TTM, the rate of survival-to-hospital discharge increased from $25.9 \%$ in 2006 to $44.0 \%$ in 2017 . As to neurological recovery at hospital discharge, the incidence of good neurological function (CPC: $1-2$ ) increased from $10.3 \%$ in 2006 to $36.7 \%$ in 2017 . The rates of survival and good neurological outcome each year were shown in Figure 3.

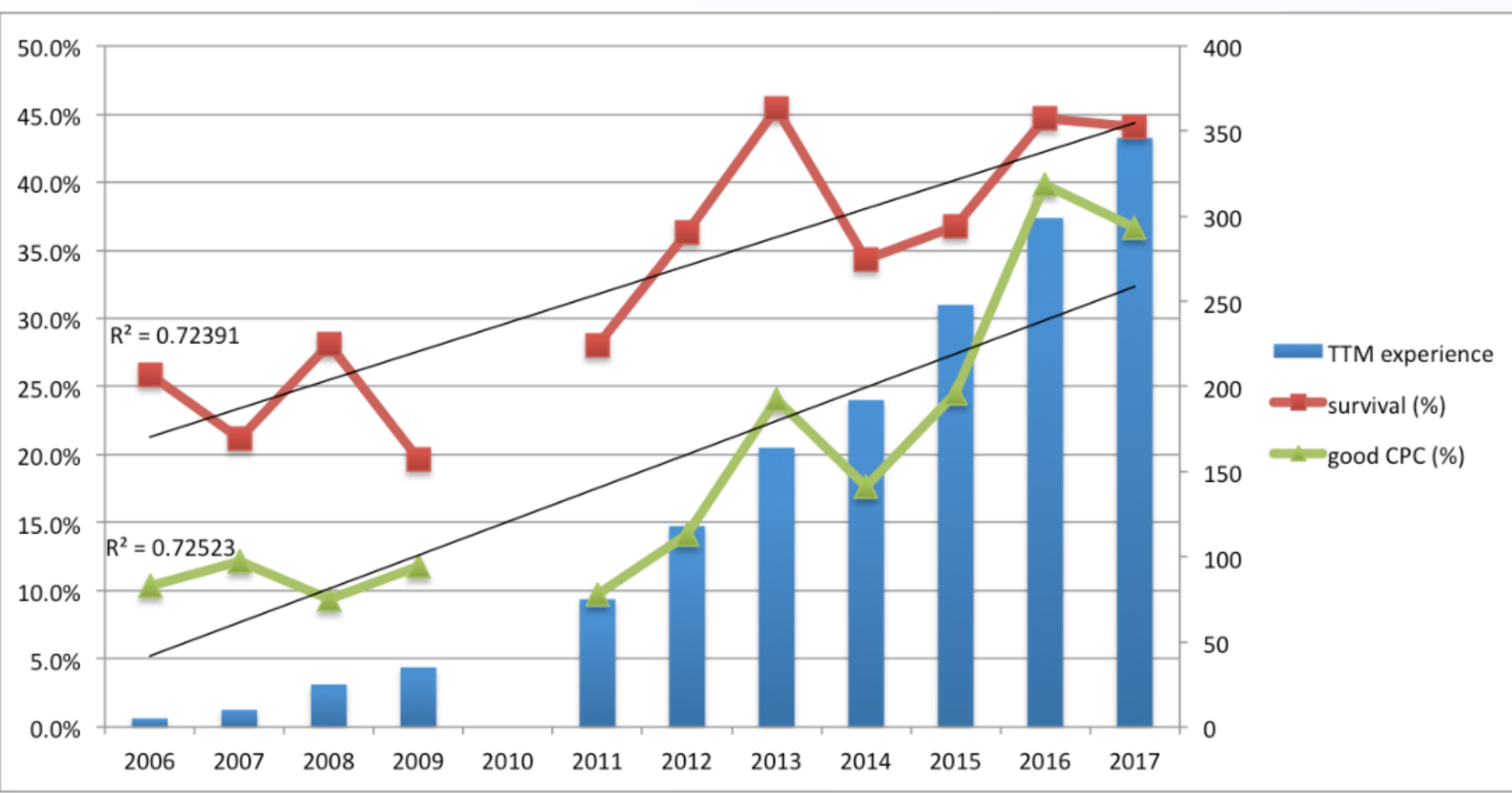

Figure 3. The rates of survival and good neurological outcome and accumulated TTM experience per year.

Multiple logistic regression demonstrated significant association between TTM accumulated experience and both survival (odds ratio $1.002,95 \% \mathrm{Cl} 1.000-1.003$ ) and neurological outcome (odds ratio $1.005,95 \% \mathrm{Cl} 1.003-1.007)$ in the non-TTM group.

\section{Conclusions}

The accumulation of TTM experience is associated with improved neurological recovery in cardiac arrest survivors not receiving temperature management. 